Review

\title{
Imaging Nanotherapeutics in Inflamed Vasculature by Intravital Microscopy
}

\author{
Zhenjia Wang ${ }^{凶}$ \\ Department of Pharmaceutical Sciences, College of Pharmacy, Washington State University, Spokane, WA, USA \\ $\bowtie$ Corresponding author: zhenjia.wang@wsu.edu
}

(C) Ivyspring International Publisher. Reproduction is permitted for personal, noncommercial use, provided that the article is in whole, unmodified, and properly cited. See http://ivyspring.com/terms for terms and conditions.

Received: 2016.05.27; Accepted: 2016.08.04; Published: 2016.10.08

\begin{abstract}
Intravital microscopy (IVM) is the application of light microscopy to real time study biology of live animal tissues in intact and physiological conditions with the high spatial and temporal resolution. Advances in imaging systems, genetic animal models and imaging probes, IVM has offered quantitative and dynamic insight into cell biology, immunology, neurobiology and cancer. In this review, we will focus on the targeting of nanotherapeutics to inflamed vasculature. We will introduce the basic concept and principle of IVM and demonstrate that IVM is a powerful tool used to quantitatively determine the molecular mechanisms of interactions between nanotherapeutics and neutrophils or endothelium in living mice. In the future, it is needed to develop new imaging systems and novel imaging contrast agents to better understand molecular mechanisms of tissue processing of nanotherapeutics in vivo.
\end{abstract}

Key words: Nanoparticles, Intravital Microscopy (IVM), Inflammation, Neutrophils and Endothelium.

\section{Introduction}

Nanomedicines are conceptually defined as nanotechnology applications in medicine including biosensing[1], diagnostics[2, 3], imaging[4, 5] and targeted drug delivery [4, 6-9]. Targeted drug delivery using nanoparticles could dramatically increase therapeutic efficacy and avoid the systemic toxicity $[10,11]$. When nanoparticle drug carriers are administrated to animals or patients, it is necessary to know the pharmacodynamics of nanoparticles in circulation, and how nanoparticles temporally interact with targeted tissues at a molecular level. Traditionally, tissues are fixed and then histochemically stained for imaging using fluorescence confocal microscopy to determine the subcellular locations of nanoparticles. Nanoparticles are totally different from molecules (such as drugs) in size, shape, surface charges and chemical composition, and they are highly heterogeneous, therefore tissue processing of nanoparticles is complex and dynamic $[12,13]$. Direct imaging of live animals at a high spatial and temporal resolution would address dynamic interactions between nanoparticles and tissues at a subcellular level. To do so, this requires to develop advanced imaging systems which are able to real-time record the temporal binding, internalization and cellular trafficking of nanoparticles in live tissues under physiological or pathological conditions.

Due to recent advances in imaging hardware and software, laser fluorescence microscopy has been applied to imaging live mammalian tissues. This new imaging approach is called intravital microscopy (IVM) [14-16]. IVM has rapidly become an essential tool in studying neurobiology [17, 18], immunology $[19,20]$, molecular biology [21] and cancer biology [22, 23]. There are several comprehensive review articles covering these topics [14, 20, 23], but few reviews have discussed the application of IVM in imaging therapeutic nanoparticles in inflamed vasculatures. When combined with pharmacological approaches (knockout mouse models) and powerful imaging software, IVM could quantitatively address the 
dynamic interactions between nanoparticles and live tissues at a subcellular resolution, thus revealing the insight of tissue processing of nanoparticles. In this short review, we will focus on the recent developments on IVM applied in understanding how therapeutic nanoparticles specifically target inflamed sites. As well we will introduce a principle of IVM, and demonstrate the power and usefulness of IVM in targeted drug delivery using nanoparticles.

\section{Intravital Microscopy (IVM)}

Fig. 1 shows a concept of IVM in imaging cremaster muscles of a live mouse [24] (Fig. 1A) and nanovesicles made from activated neutrophil membrane are adherent to endothelium in cremaster venules imaged by IVM [7] (Fig. 1B). Fig. 1 shows that we need three components to build IVM. A microscope is used to image fluorescently-labeled nanoparticles (such as nanovesicles) in the live tissues of interest (such as cremaster muscles). Therefore, constructing IVM requires several disciplines, such as, optics, biology and materials engineering.

IVM is composed of three major technologies. The first technology is a light-based microscope. A fluorescence microscope is commonly used to build IVM, such as wide-field fluorescence microscopy, laser scanning confocal microscopy, laser multiphoton scanning confocal microscopy, and spinning disk confocal microscopy. IVM needs the low incident light to prevent damage of tissues or fluorophores, and also requires a spatial and temporal resolution sufficient to observe a single cell. Fluorescence confocal microscopy (laser scanning or spinning-disk models) as shown in Fig. 1A is commonly used in IVM because of the low cost and easy use, but a major challenge is the limitation of visible light penetration in tissues. Multiphoton fluorescence-based IVM microscopy allows to observe deeper tissues due to the laser excitation in near infrared wavelengths. Multiphoton microscopy [25] is a nonlinear optical process that requires the laser beam concentrating in a very small focal point. This results in less photobleaching, lower phototoxicity, and the reduction of auto-fluorescence compared to regular fluorescence confocal microscopes. However, the maintenance of a multiphoton microscope is very high. Other microscope technologies could be chosen to expand a wide range of applications of IVM. For example, fluorescence life time microscopy [26], coherent anti-stokes Raman microscopy (CARS) [27] and optical frequency domain imaging [28] have been used in IVM. As well, surface-enhanced Raman spectroscopy could be incorporated into IVM [29, 30]. Here we focus on the discussion of fluorescence-based IVM.
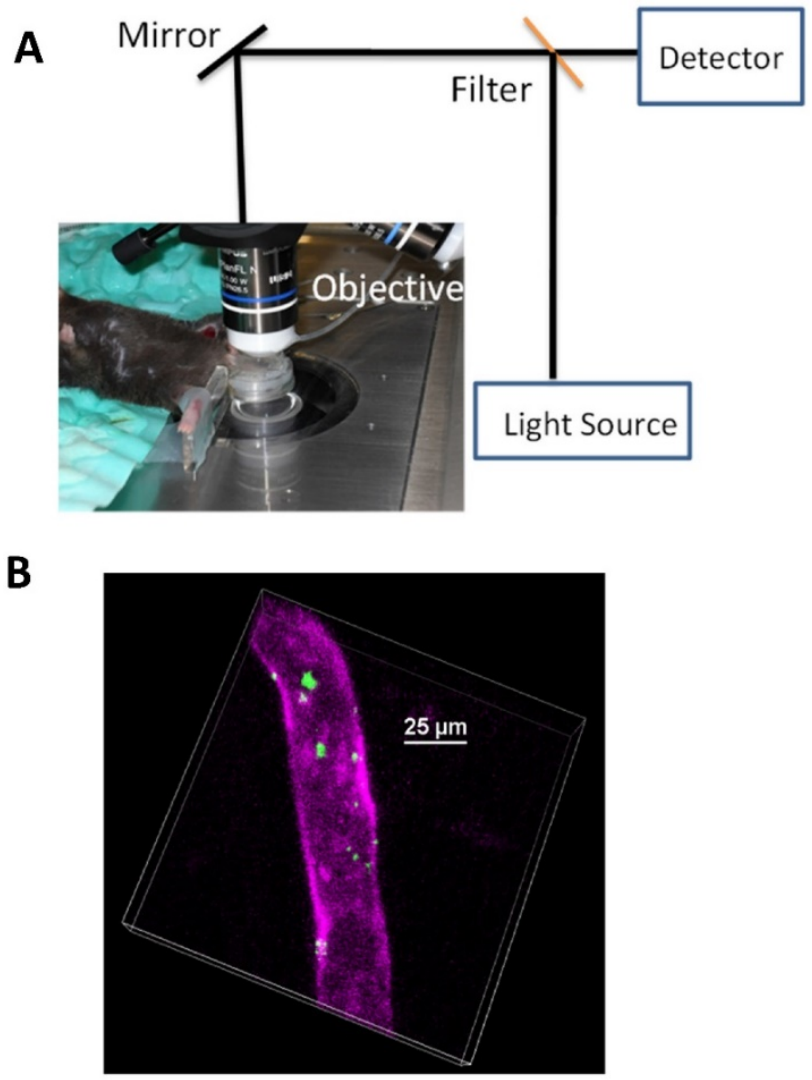

Figure 1. (a) Setup of IVM of cremaster post-capillary venules in a live mouse. The cremaster muscles are surgically exposed and perfused with a physiological buffer under an objective, and images are recorded using laser scanning confocal microscope or spinning-disc confocal microscope. (b) 3D image of IVM shows the adhesion of neutrophil membrane-formed nanovesicles to endothelium $3 \mathrm{~h}$ after introscrotal injection of TNF- $\alpha$. The nanovesicles were labeled by $\mathrm{DiO}$ dyes (green) and the vessel was stained with Alexa-Fluor-647-anti-CD31 (pink). The image $B$ is reproduced and permitted from the reference [7].

The second component of IVM is the development of suitable mouse models in which biological questions can be answered within a setting that is not only physiological relevant but also accessible to observe organs of interest. For example, the dorsal skin window chamber model was developed several decades ago, but still remains helpful in cancer biology [31]. A mouse implanted with a window chamber can be maintained for several weeks, allowing to image live tissues over a long period. To investigate the interactions between leukocytes and endothelium, or tissue homeostasis, cremaster muscle tissues are usually used [24] (Fig. 1B). IVM of cremaster tissues is useful and easy to observe the microcirculation because a thin and transparent layer of cremaster tissue is spread under a water-immersion objective. A steady drip of sodium buffer is maintained throughout the imaging period so cremaster tissues are kept under a physiological condition. Other organs can be accessible even though surgical procedures with variable degrees of invasiveness. For example, the liver is exteriorized 
and superfused with a physiological saline solution for imaging [32]. The liver is an important and critical component in the immune defense against blood-born infection because the liver contains innate and adaptive immune cells which can detect and capture pathogens from the blood. IVM of the liver offers the quantitative and dynamic details of the host defense [33]. The lung is a unique organ with an air-liquid interface exposing it to the environment and with a large volume of blood that quickly circulates in the lung. The lung microcirculation is also distinctive with a rich network of capillary segments with a diameter as small as a few microns, which requires many leukocytes to deform their shape to pass through. The novel feature of the lung is needed to directly image, however this demands fast, high-resolution, and penetrant imaging methodologies as well as maintenance of intact physiology. The lung movement is a particular problem for imaging because of cardiac contractions, pulsatile blood flow, and significant movements during inhalation/exhalation cycle. A thoracic window was built to stabilize the lung movement [34]. An important feature of this device is to loosen adherence to the lung surface via $20-25 \mathrm{mmHg}$ of reversible vacuum spreading over a large local area allowing for gas to enter and expand the lung, therefore this limits the associated movement of lung. IVM of the stabilized lung reveals the novel properties of lung cancer metastasis via tumor microparticles (microvesicles) and immune defense, which cannot be observed using conventional imaging systems [35].

The third technology of IVM is to develop new imaging probes [36]. The majority of IVM studies use fluorescent proteins and exogenous fluorophores to generate the image contrast. To visualize deep tissues, bright and stable near-infrared fluorescent proteins have been developed [37, 38]. A large number of genetically encoded fluorescent probes with the cleavable or photoactivated/switchable features are under development. To increase signal-to-noise ratio, nanoparticles conjugated with antibodies are also used in IVM, such as quantum dots [39]. To visualize therapeutic nanoparticles in vivo using IVM, the nanoparticles are fluorescently labeled and can emit different colors.

\section{Inflammation and Neutrophils}

Inflammation is a normal response of innate and adaptive immune systems to infection and tissue damages [40]. However, when allowed to continue uncontrolled, inflammation will become non-resolved, thus resulting in a wide range of diseases, such as autoimmune or autoinflammatory disorders, neurodegenerative disease or cancers
[41-44]. Therefore, impairing inflammation pathways or resolving inflammation [45] using nanotherapeutics would be a means to prevent the inflammation-related diseases [24].

Neutrophils, a type of polymorphonuclear leukocytes, are well known as a major player during acute inflammation $[46,47]$. However, several studies suggest that neutrophils also play a significant role in both initiating and shaping the immune response to many chronic inflammatory diseases, such as atherosclerosis [48, 49], autoimmune disease [50], and even cancer [51]. Neutrophils are typically the first leukocytes to be recruited to an inflammatory site and are capable of eliminating pathogens by three mechanisms [52] (phagocytosis, degranulation and neutrophil extracellular traps, NETs). Data from experimental and clinical settings show that following infection or tissue damages, the recruitment of neutrophils to the site of inflammation is critical. In this process, macrophages and mast cells reside in tissues and act as sentinel cells that initiate neutrophil recruitment via several steps including rolling, adhesion, crawling and endothelial transmigration. The interaction between neutrophils and vascular endothelium is a primary component in the inflammation response. Neutrophil binding and adhesion to endothelium require integrin proteins (such as $\beta_{2}$ ) on neutrophils interacting with intercellular adhesion molecule-1 (ICAM-1) on endothelium. Direct imaging of neutrophil-endothelium interactions in intact pathological conditions would help to develop novel strategies to facilitate the delivery of nanotherapeutics to the site of vascular inflammation, thus diminishing excessive inflammation responses that cause a wide range of diseases, such as acute lung injury, sepsis and cancer.

\section{Nanoparticle Targeting of Activated Neutrophils}

Neutrophil adhesion to activated endothelium and subsequently endothelial transmigration are essential processes in innate immune response that eliminates pathogens to promote bacterial clearance $[52,53]$. Neutrophil recruitment to the site of tissue injury is the first line of host defense, but dysregulated activation and infiltration of neutrophils at the vessel wall are also a major cause of inflammation-related diseases, such as acute lung injury, sepsis and ischemia-reperfusion injury [54-56]. Targeted drug delivery to activated neutrophils is a promising strategy to impair excessive inflammation to reverse the progression of the diseases. The studies have shown that inhibiting integrin $\beta_{2}$ highly expressed on activated neutrophils by anti-integrin $\beta_{2}$ 
antibodies can diminish the adhesion of neutrophils to endothelial cells, thus leading to the restoration of vascular integrity [57], implying that deactivation of neutrophils would be a novel strategy to prevent acute vascular inflammation.

Advances in nanotechnology and imaging systems enable to image dynamic interactions between neutrophils and nanoparticles in vivo. The recent studies using IVM showed that an approach of employing drug-loaded albumin nanoparticles, can efficiently deliver drugs into neutrophils adherent to activated endothelium [24]. To demonstrate albumin nanoparticle targeting of activated neutrophils, IVM of mouse cremaster post-capillary venules was performed to real time visualize the interactions of albumin nanoparticles with neutrophils. In the experiments, albumin nanoparticles [58] $(100 \mathrm{~nm}$ in diameter) were labeled with $\mathrm{Cy} 5$ fluorescent dyes and neutrophils were stained with Alexa Fluor 488 anti-mouse Gr-1 antibody, then live cremaster venules were imaged using IVM. Intravital images showed the marked co-localization of anti-Gr-1 antibody and albumin nanoparticles (Fig. 2A), illustrating that albumin nanoparticles were internalized by neutrophils. Without TNF-a treatment (no

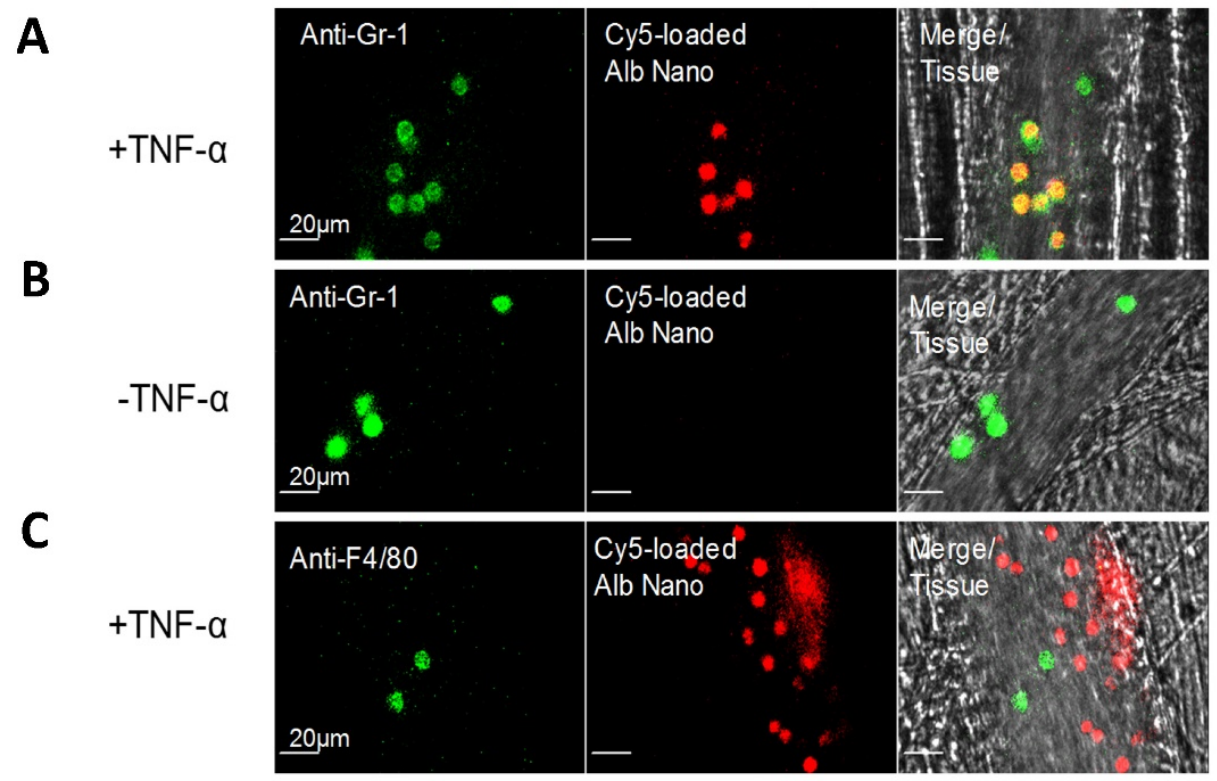

D

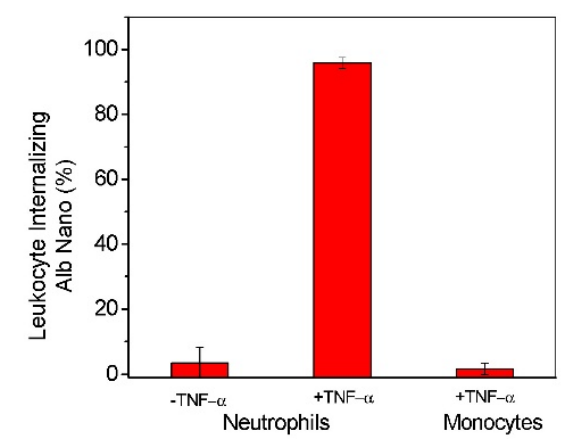

inflammation), resting (or circulating) neutrophils did not take up albumin nanoparticles (Fig. 2B) [24]. The result suggests that albumin nanoparticles can selectively target activated neutrophils, which allows the precise targeting of nanoparticles to inflammation sites.

In acute inflammation, monocytes, another type of phagocytic cells, in the bloodstream could be activated to take up albumin nanoparticles [59]. To examine whether activated monocytes took up albumin nanoparticles, Alexa Fluor 488-labeled anti-mouse F4/80 antibody (a marker for monocytes) [59] and albumin nanoparticles were intravenously infused into TNF-a-challenged mice, IVM images showed no co-staining between monocytes and nanoparticles (Fig. 2C), indicating that activated monocytes do not take up albumin nanoparticles. While IVM imaging qualitatively demonstrates the uptake of nanoparticles by activated neutrophils, the quantitative analysis of images is critical to draw the solid conclusions. In the paper, the authors [24] have quantitatively analyzed the co-staining between albumin nanoparticles and neutrophils or monocytes by counting objects and obtained the percentage of their co-staining. The result shows that more than $90 \%$ of activated neutrophils take up nanoparticles, but it is unlike that nanoparticles are taken up by resting neutrophils or activated monocytes (Fig. 2D).

Figure 2. IVM demonstrates the uptake of albumin nanoparticles by activated neutrophils in live cremaster venules. Cy5-loaded albumin nanoparticles (red) are internalized by activated neutrophils following intrascrotal injection of TNF- $\alpha$ (a) and without injection of TNF- $\alpha$ (b) in mice. Neutrophils (green) were visualized by intravenous infusion of Alexa Fluor-488 anti-mouse Gr-1 antibody. In the TNF- $\alpha$-challenged group, the nanoparticles were intravenously infused $3 \mathrm{~h}$ post-intrascrotal injection of TNF- $\alpha$. (c) Monocytes (green) were visualized after infusion of Alexa Fluor-488 anti-mouse F4/80 antibody $3 \mathrm{~h}$ after TNF- $\alpha$-injection. Scale bar, $20 \mu \mathrm{m}$. (d) Percentage of neutrophils and monocytes internalizing albumin nanoparticles. All data represent means \pm s.e.m ( $n=13-20$ vessels in three mice per group). The images are reproduced and permitted from the reference [24]. 
Neutrophil uptake of albumin nanoparticles is very rapid after intravenous administration, so the uptake process should be mediated by receptors highly expressed on neutrophils. IVM was applied to study the uptake efficiency of different types of albumin nanoparticles (such as albumin-coated polystyrene nanoparticles) and their uptake in genetic knockout mice (such as knockout Fcy receptors and integrin $\beta_{2}$ on neutrophils). It is interesting to find that denatured albumin protein after the formation of nanoparticles binds to $F_{C \gamma}$ receptors on activated neutrophils to mediate the nanoparticle uptake [24]. This result is consistent with the studies where $\mathrm{Fc} \gamma$ receptors mediate the endocytosis of denatured immune complexes of protein for their clearance by the immune systems [60].

Furthermore, IVM of the cremaster model was used to investigate the therapeutic effect [24] when albumin nanoparticles were loaded with piceatannol [61], an inhibitor of Syk kinase. The Syk signaling pathway is responsible for the mechanism of outside-in integrin signaling that mediates neutrophil adhesion, spreading and migration via integrin $\beta_{2}$ dependence $[62,63]$. Piceatannol selectively inhibits Syk activity. In the study, intrascrotal injection of TNF-a caused local vascular inflammation which showed the adherence of neutrophils to cremaster venuels when they were imaged by IVM (Fig. 3A). Subsequently, piceatannol-loaded albumin nanoparticles were intravenously infused to the same mouse. In a short period $(30 \mathrm{~min})$ the adherent neutrophils began to move and roll along venular walls and some of the adherent neutrophils detached from venular walls (Fig. 3B). IVM movies clearly demonstrated therapeutic effects of drug-loaded albumin nanoparticles. Most importantly, IVM images can be used to quantitatively analyze the properties of neutrophil adhesion and rolling to evaluate therapeutic effects after administration of drug-loaded albumin nanoparticles (Fig. 3C and D). The IVM provides convincing results to validate the hypothesis. Most importantly, therapeutic effects of drug-loaded nanoparticles can be real-time measured and quantitatively analyzed in intact diseased mouse models.
A
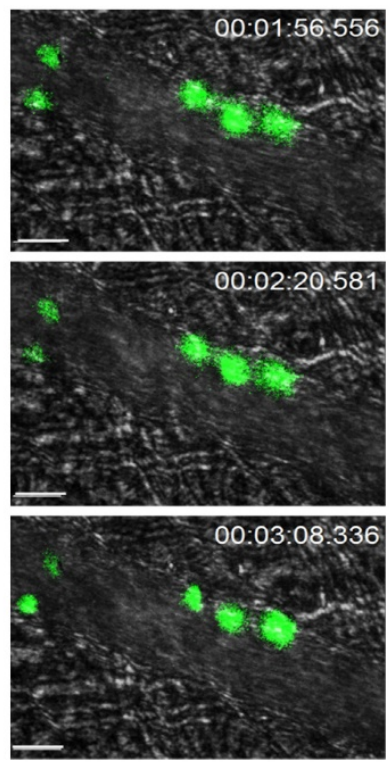

C

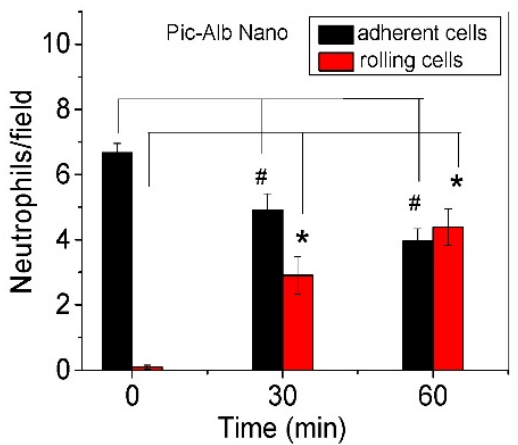

B
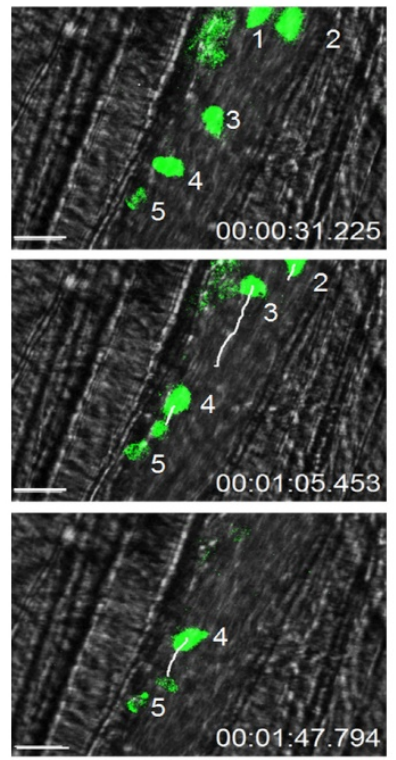

D

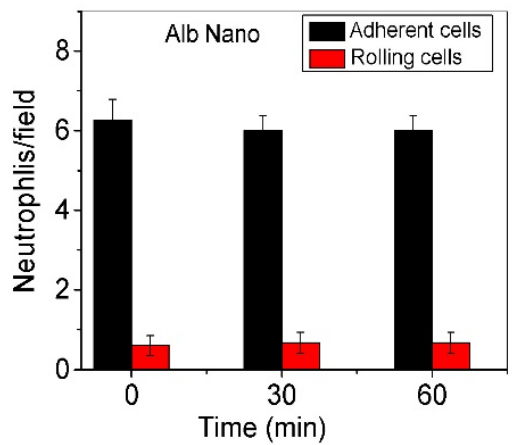

Figure 3. Therapeutic effects of drug-loaded albumin nanoparticles are evaluated using IVM. IVM of cremaster venules showing adhesion and rolling of neutrophils (green) labeled by Alexa Fluor-488 anti-mouse Gr-1 antibody in a mouse before (a) and at $1 \mathrm{~h}$ after intravenous injection of piceatannol-loaded albumin nanoparticles (b) in the same mouse. hh:mm:ss represents time series of images. The white lines show the trajectories of neutrophils detaching from endothelium. Scale bar, $20 \mu \mathrm{m}$. (c) Quantification of neutrophil adhesion and rolling in TNF- $\alpha$-activated cremaster venules at the baseline, and at 30 and $60 \mathrm{~min}$ after intravenous infusion of piceatannol-loaded albumin nanoparticles. Data represent mean \pm s.e.m $(n=21$ venules in three mice). ${ }^{*} p<0.01$ and $*_{p}<0.001$ versus pre-infusion of nanoparticles. (d) albumin nanoparticles without piceatannol were tested and quantified as described in c. Data represent mean \pm s.e.m. $(n=18$ venules in three mice). The images are reproduced and permitted from the reference [24]. 
The major challenge in nanoparticle drug delivery is how to overcome the blood vessel barrier to efficiently deliver therapeutics in deep tissues. The endothelium forms a monolayer lining the vessel wall to regulate the transport of plasma contents into tissues, and the permeability is strongly dependent on nanoparticle size. The size of nanoparticles larger than $3 \mathrm{~nm}$ are difficult to transport across the endothelial layer [64]. During acute inflammation, neutrophils transmigrate through endothelium to arrive at infected sites, and then neutrophils remove pathogens and repair the damage of tissues [52]. The trans-endothelial migration could become an active pathway to deliver drugs in disease sties if nanoparticles hijack neutrophils in vivo.

A research group [6] in Washington State University has proposed to utilize the pathway of neutrophil trans-endothelial migration to deliver nanoparticles and validated this novel concept in an acute lung inflammation mouse model. The lung has unique features with many tiny air sacs (called alveoli) surrounded by a network of blood capillaries to form an interface between circulation and airspace [65]. When infections happened in the airspace, neutrophils are capable of migrating from bloodstream to airspace passing through the endothelial vessel walls [66]. Using this model, it was observed that intravenously infused albumin nanoparticles were internalized in neutrophils and subsequently nanoparticles were accumulated in the airspace of lungs, indicating neutrophils are a cargo to deliver albumin nanoparticles across blood vessel barrier [6]. Most importantly, when neutrophils were depleted using antibodies, the transport of nanoparticles were totally prevented, illustrating that neutrophils are responsible for the nanoparticle movement. Furthermore, IVM of cremaster tissues [6] was used to real-time observe this process in which albumin nanoparticles hijacked neutrophils to transport the nanoparticles across the endothelial vessel barrier.

The examples described above demonstrate the usefulness of IVM in studying the interactions between nanotherapeutics and neutrophils in vivo at the molecular level. Combined with advanced imaging software and genetic knockout mouse models, IVM could offer the insight of molecular mechanisms at which live tissues process nanotherapeutics before they are taken up by the liver and phagocytic systems.

\section{Nanoparticle targeting of inflamed endothelium}

Inflammation usually involves mutual interactions between immune cells and vascular endothelium, which are regulated by intercellular adhesion molecules. In inflamed sites, endothelium highly expresses intercellular adhesion molecule 1 (ICAM-1) via the NF-kB pathway to recruit leukocytes [67]. To target inflamed vasculature, nanoparticles are conjugated with anti-ICAM-1 or peptides to the surface of nanoparticles [68, 69]. However, synthetic features of nanoparticles could not avoid the clearance by immune systems before the nanoparticles arrive at a target $[12,70]$.

When inflammation occurs, neutrophils, a type of polymorphonuclear leukocytes, highly express integrin $\beta_{2}$, which binds to ICAM-1 on inflamed endothelium. Recently, Wang's group [7] has proposed a novel strategy to target inflamed endothelium using cell membrane-formed nanovesicles made from activated neutrophils (differentiated HL 60 cells). The authors have developed a systemic approach to generate membrane-derived nanovesicles including cell membrane disruption by nitrogen cavitation, differential centrifugation and the size selection of nanovesicles. They quantitatively measured DNA and proteins at each centrifugation step, and studied the properties of nanovesicles using cryo-TEM and dynamic light scattering, finding that nanovesicles are mainly made from plasma membrane and the size of nanovesicles is $150 \mathrm{~nm}$ in diameter [7].

To demonstrate that neutrophil membrane-formed nanovesicles can selectively target activated endothelium, IVM was performed in mouse cremaster venuels challenged by TNF-a. Fig. 1B shows that nanovesicles were strongly adherent to activated endothelium. In a 2-3 h period of imaging, the adherent nanovesicles rarely detached from vessel walls, implying that the adherent nanovesicles were likely to be internalized by endothelial cells. Some puncta were larger than the physical size of nanovesicles, which might be associated with the infusion of nanovesicles with large organelles in the endothelium after nanovesicle internalization. It could be also associated with multiple nanovesicles closely adherent to endothelium due to the heterogeneous distribution of ICAM-1. When compared with the nanovesicles made from red blood cells which do not express integrin $\beta_{2}$, the nanovesicles do not show the endothelial adhesion when observed by IVM of mouse cremaster venules. IVM also demonstrates that activation of endothelium is required for the adhesion of neutrophil nanovesicles, indicating that the binding partner of integrin $\beta_{2}$ and ICAM-1 is the molecular mechanism for targeting of neutrophil nanovesicles to inflamed endothelium [7]. The studies show that IVM is a powerful tool to investigate the interaction between 
nanoparticles and endothelial vessels in vivo, uncovering novel features unobserved in vitro.

\section{Conclusion and Perspectives}

In this review, we have shown the capability and usefulness of IVM applied to studying the internalization of nanotherapeutics by neutrophils or endothelium in vivo. We also demonstrate that IVM can quantitatively address molecular mechanisms of tissue processing of nanotherapeutics and their therapeutic effects using advanced imaging software and genetic approaches. Nanoparticle targeted drug delivery is a new field in pharmaceutical sciences, biology and medicine. Tissue processing of nanotherapeutics is complicated and dynamics. To real time track this interaction, it is urgent to develop novel and advanced imaging systems, such as fluorescence-based or Raman scattering-based IVM. It is also needed to make bright and different colors of nanoparticles. To study the trafficking of nanotherapeutics in sub-cellular components, the nanoparticles could be fluorescently labeled by $\mathrm{pH}$ sensitive probes or other imaging components. Genetic and pharmacological approaches will be needed to develop and combine with IVM to address molecular mechanisms regulating binding, internalization and transport of nanoparticles in a single cell (such as immune cells or endothelium). With the advances in imaging systems and software, we believe that IVM would be an essential tool in nanomedicines, cancer biology and immunology. Specifically, evaluating nanotherapeutics in intact pathological conditions using IVM would shift the current paradigm of drug discovery and nanoparticle drug formulations.

\section{Acknowledgement}

The work is supported by NIH grants K25HL111157 and RO1GM116823.

\section{Competing Interests}

The author has declared no competing interest exists.

\section{References}

1. Zhang Y, Huang L, Li Z, Ma G, Zhou Y, Han G. Illuminating Cell Signaling with Near-Infrared Light-Responsive Nanomaterials. ACS Nano. 2016; 10: 3881-5.

2. Melemenidis S, Jefferson A, Ruparelia N, Akhtar AM, Xie J, Allen D, et al. Molecular magnetic resonance imaging of angiogenesis in vivo using polyvalent cyclic RGD-iron oxide microparticle conjugates. Theranostics. 2015; 5. 515-29.

3. Xie J, Liu G, Eden HS, Ai H, Chen X. Surface-engineered magnetic nanoparticle platforms for cancer imaging and therapy. Acc Chem Res. 2011; 44: 883-92.

4. Wang Z, Tiruppathi C, Minshall RD, Malik AB. Size and dynamics of caveolae studied using nanoparticles in living endothelial cells. ACS Nano. 2009; 3: 4110-6.

5. $\mathrm{Yu} \mathrm{M}$, Zheng J. Clearance Pathways and Tumor Targeting of Imaging Nanoparticles. ACS Nano. 2015; 9: 6655-74
6. Chu D, Gao J, Wang Z. Neutrophil-Mediated Delivery of Therapeutic Nanoparticles across Blood Vessel Barrier for Treatment of Inflammation and Infection. ACS Nano. 2015; 9: 11800-11.

7. Gao J, Chu D, Wang Z. Cell membrane-formed nanovesicles for disease-targeted delivery. J Control Release. 2016; 224: 208-16.

8. Wang Z. Caveolae-mediated Delivery of Therapeutic Nanoparticles across Blood-endothelial Barrier. Austin J Anal Pharm Chem. 2014; 1.

9. Wang Z, Tiruppathi C, Cho J, Minshall RD, Malik AB. Delivery of nanoparticle: complexed drugs across the vascular endothelial barrier via caveolae. IUBMB Life. 2011; 63: 659-67.

10. Farokhzad OC, Langer R. Impact of nanotechnology on drug delivery. ACS Nano. 2009; 3: 16-20

11. Mitragotri S, Burke PA, Langer R. Overcoming the challenges in administering biopharmaceuticals: formulation and delivery strategies. Nat Rev Drug Discov. 2014; 13: 655-72.

12. Cheng CJ, Tietjen GT, Saucier-Sawyer JK, Saltzman WM. A holistic approach to targeting disease with polymeric nanoparticles. Nat Rev Drug Discov. 2015; 14: 239-47.

13. Park K. Controlled drug delivery systems: past forward and future back. J Control Release. 2014; 190: 3-8.

14. Pittet MJ, Weissleder R. Intravital imaging. Cell. 2011; 147: 983-91.

15. Masedunskas A, Milberg O, Porat-Shliom N, Sramkova M, Wigand T, Amornphimoltham $\mathrm{P}$, et al. Intravital microscopy: a practical guide on imaging intracellular structures in live animals. Bioarchitecture. 2012; 2: 143-57.

16. Masedunskas A, Porat-Shliom N, Rechache K, Aye MP, Weigert R. Intravital Microscopy Reveals Differences in the Kinetics of Endocytic Pathways between Cell Cultures and Live Animals. Cells. 2012; 1: 1121-32.

17. Pai S, Danne KJ, Qin J, Cavanagh LL, Smith A, Hickey MJ, et al. Visualizing leukocyte trafficking in the living brain with 2-photon intravital microscopy. Front Cell Neurosci. 2012; 6: 67.

18. Yang G, Pan F, Gan WB. Stably maintained dendritic spines are associated with lifelong memories. Nature. 2009; 462: 920-4

19. Friedman RS, Beemiller P, Sorensen CM, Jacobelli J, Krummel MF. Real-time analysis of $\mathrm{T}$ cell receptors in naive cells in vitro and in vivo reveals flexibility in synapse and signaling dynamics. J Exp Med. 2010; 207: 2733-49.

20. Krummel MF. Illuminating emergent activity in the immune system by real-time imaging. Nat Immunol. 2010; 11: 554-7.

21. Masedunskas A, Sramkova M, Parente L, Sales KU, Amornphimoltham P, Bugge $\mathrm{TH}$, et al. Role for the actomyosin complex in regulated exocytosis revealed by intravital microscopy. Proc Natl Acad Sci U S A. 2011; 108: $13552-7$.

22. Ellenbroek SI, van Rheenen J. Imaging hallmarks of cancer in living mice. Nat Rev Cancer. 2014; 14: 406-18.

23. Jain RK, Munn LL, Fukumura D. Dissecting tumour pathophysiology using intravital microscopy. Nat Rev Cancer. 2002; 2: 266-76.

24. Wang Z, Li J, Cho J, Malik AB. Prevention of vascular inflammation by nanoparticle targeting of adherent neutrophils. Nat Nanotechnol. 2014; 9: 204-10.

25. Entenberg D, Wyckoff J, Gligorijevic B, Roussos ET, Verkhusha VV, Pollard JW, et al. Setup and use of a two-laser multiphoton microscope for multichannel intravital fluorescence imaging. Nat Protoc. 2011; 6: 1500-20.

26. Provenzano PP, Eliceiri KW, Keely PJ. Multiphoton microscopy and fluorescence lifetime imaging microscopy (FLIM) to monitor metastasis and the tumor microenvironment. Clin Exp Metastasis. 2009; 26: 357-70.

27. Fu Y, Huff TB, Wang HW, Wang H, Cheng JX. Ex vivo and in vivo imaging of myelin fibers in mouse brain by coherent anti-Stokes Raman scattering microscopy. Opt Express. 2008; 16: 19396-409.

28. Vakoc BJ, Lanning RM, Tyrrell JA, Padera TP, Bartlett LA, Stylianopoulos T, et al. Three-dimensional microscopy of the tumor microenvironment in vivo using optical frequency domain imaging. Nat Med. 2009; 15: 1219-23.

29. Wang Z, Pan S, Krauss TD, Du H, Rothberg LJ. The structural basis for giant enhancement enabling single-molecule Raman scattering. Proc Natl Acad Sci US A. 2003; 100: 8638-43.

30. Wang Z, Rothberg LJ. Origins of blinking in single-molecule Raman spectroscopy. J Phys Chem B. 2005; 109: 3387-91.

31. Brown EB, Campbell RB, Tsuzuki Y, Xu L, Carmeliet P, Fukumura D, et al. In vivo measurement of gene expression, angiogenesis and physiological function in tumors using multiphoton laser scanning microscopy. Nat Med. 2001; 7: 864-8.

32. Cools-Lartigue J, Spicer J, McDonald B, Gowing S, Chow S, Giannias B, et al. Neutrophil extracellular traps sequester circulating tumor cells and promote metastasis. J Clin Invest. 2013.

33. Jenne CN, Kubes P. Immune surveillance by the liver. Nat Immunol. 2013; 14 : 996-1006.

34. Looney MR, Thornton EE, Sen D, Lamm WJ, Glenny RW, Krummel MF. Stabilized imaging of immune surveillance in the mouse lung. Nat Methods. 2011; 8: 91-6.

35. Headley MB, Bins A, Nip A, Roberts EW, Looney MR, Gerard A, et al. Visualization of immediate immune responses to pioneer metastatic cells in the lung. Nature. 2016; 531: 513-7.

36. Marx V. Optimizing probes to image cleared tissue. Nat Methods. 2016; 13: 205-9. 
37. Filonov GS, Krumholz A, Xia J, Yao J, Wang LV, Verkhusha VV. Deep-tissue photoacoustic tomography of a genetically encoded near-infrared fluorescent probe. Angew Chem Int Ed Engl. 2012; 51: 1448-51.

38. Filonov GS, Piatkevich KD, Ting LM, Zhang J, Kim K, Verkhusha VV. Bright and stable near-infrared fluorescent protein for in vivo imaging. Nat Biotechnol. 2011; 29: 757-61.

39. Smith BR, Cheng Z, De A, Koh AL, Sinclair R, Gambhir SS. Real-time intravital imaging of RGD-quantum dot binding to luminal endothelium in mouse tumor neovasculature. Nano Lett. 2008; 8: 2599-606.

40. Medzhitov R. Inflammation 2010: new adventures of an old flame. Cell. 2010; 140: 771-6.

41. Dinarello CA. Anti-inflammatory Agents: Present and Future. Cell. 2010; 140: 935-50.

42. Grivennikov SI, Greten FR, Karin M. Immunity, inflammation, and cancer. Cell. 2010; 140: 883-99.

43. Nathan C, Ding A. Nonresolving inflammation. Cell. 2010; 140: 871-82.

44. Kastner DL, Aksentijevich I, Goldbach-Mansky R. Autoinflammatory disease reloaded: a clinical perspective. Cell. 2010; 140: 784-90.

45. Serhan CN, Petasis NA. Resolvins and protectins in inflammation resolution. Chem Rev. 2011; 111: 5922-43.

46. Kolaczkowska E, Kubes P. Neutrophil recruitment and function in health and inflammation. Nat Rev Immunol. 2013; 13: 159-75.

47. Mayadas TN, Cullere X, Lowell CA. The multifaceted functions of neutrophils. Annu Rev Pathol. 2014; 9: 181-218.

48. Ionita MG, van den Borne P, Catanzariti LM, Moll FL, de Vries JP, Pasterkamp $\mathrm{G}$, et al. High neutrophil numbers in human carotid atherosclerotic plaques are associated with characteristics of rupture-prone lesions. Arterioscler Thromb Vasc Biol. 2010; 30: 1842-8.

49. Drechsler M, Megens RT, van Zandvoort M, Weber C, Soehnlein O. Hyperlipidemia-triggered neutrophilia promotes early atherosclerosis. Circulation. 2010; 122: 1837-45.

50. Kallenberg CG, Heeringa P, Stegeman CA. Mechanisms of Disease: pathogenesis and treatment of ANCA-associated vasculitides. Nat Clin Pract Rheumatol. 2006; 2: 661-70.

51. Fridlender ZG, Albelda SM. Tumor-associated neutrophils: friend or foe? Carcinogenesis. 2012; 33: 949-55.

52. Phillipson M, Kubes $\mathrm{P}$. The neutrophil in vascular inflammation. Nat Med. 2011; 17: 1381-90.

53. Wong $\mathrm{CH}$, Heit $\mathrm{B}$, Kubes P. Molecular regulators of leucocyte chemotaxis during inflammation. Cardiovasc Res. 2010; 86: 183-91.

54. Matthay MA, Zemans RL. The acute respiratory distress syndrome: pathogenesis and treatment. Annu Rev Pathol. 2011; 6: 147-63.

55. Tabas I, Glass CK. Anti-inflammatory therapy in chronic disease: challenges and opportunities. Science. 2013; 339: 166-72.

56. Wagner DD, Frenette PS. The vessel wall and its interactions. Blood. 2008; 111: 5271-81.

57. Lockwood CM, Elliott JD, Brettman L, Hale G, Rebello P, Frewin M, et al. Anti-adhesion molecule therapy as an interventional strategy for autoimmune inflammation. Clin Immunol. 1999; 93: 93-106.

58. Weber C, Kreuter J, Langer K. Desolvation process and surface characteristics of HSA-nanoparticles. Int J Pharm. 2000; 196: 197-200.

59. Sumagin R, Prizant H, Lomakina E, Waugh RE, Sarelius IH. LFA-1 and Mac-1 define characteristically different intralumenal crawling and emigration patterns for monocytes and neutrophils in situ. J Immunol. 2010; 185: 7057-66.

60. Chen K, Nishi H, Travers R, Tsuboi N, Martinod K, Wagner DD, et al. Endocytosis of soluble immune complexes leads to their clearance by FcgammaRIIIB but induces neutrophil extracellular traps via FcgammaRIIA in vivo. Blood. 2012; 120: 4421-31.

61. Geahlen RL, McLaughlin JL. Piceatannol (3,4,3',5'-tetrahydroxy-trans-stilbene) is a naturally occurring protein-tyrosine kinase inhibitor. Biochem Biophys Res Commun. 1989; 165: 241-5.

62. Evans R, Lellouch AC, Svensson L, McDowall A, Hogg N. The integrin LFA-1 signals through ZAP-70 to regulate expression of high-affinity LFA-1 on T lymphocytes. Blood. 2011; 117: 3331-42.

63. Zarbock A, Lowell CA, Ley K. Spleen tyrosine kinase Syk is necessary for E-selectin-induced alpha(L)beta(2) integrin-mediated rolling on intercellular adhesion molecule-1. Immunity. 2007; 26: 773-83.

64. Mehta D, Malik AB. Signaling mechanisms regulating endothelial permeability. Physiol Rev. 2006; 86: 279-367.

65. Ware LB, Matthay MA. Clinical practice. Acute pulmonary edema. N Engl J Med. 2005; 353: 2788-96.

66. Matthay MA, Ware LB, Zimmerman GA. The acute respiratory distress syndrome. J Clin Invest. 2012; 122: 2731-40.

67. Strnad J, Burke JR. IkappaB kinase inhibitors for treating autoimmune and inflammatory disorders: potential and challenges. Trends Pharmacol Sci. 2007; 28: $142-8$

68. Muro S, Dziubla T, Qiu W, Leferovich J, Cui X, Berk E, et al. Endothelial targeting of high-affinity multivalent polymer nanocarriers directed to intercellular adhesion molecule 1. J Pharmacol Exp Ther. 2006; 317: 1161-9.

69. Khondee S, Baoum A, Siahaan TJ, Berkland C. Calcium condensed LABL-TAT complexes effectively target gene delivery to ICAM-1 expressing cells. Mol Pharm. 2011; 8: 788-98.

70. Salvati A, Pitek AS, Monopoli MP, Prapainop K, Bombelli FB, Hristov DR, et al. Transferrin-functionalized nanoparticles lose their targeting capabilities when a biomolecule corona adsorbs on the surface. Nat Nanotechnol. 2013; 8 . $137-43$. 